\title{
Modified Visual Magnetic Resonance Rating Scale for Evaluation of Patients with Forgetfulness
}

\author{
Betul Z. Yalciner, Melek Kandemir, Sencan Taskale, Savas M. Tepe, Devrim Unay
}

\begin{abstract}
Background: As cognitive impairment increases with age, sulcal atrophy (SA) and the enlargement of the ventricles also increase. Considering the measurements on the previously proposed visual scales, a new scale is proposed in this study that allows us to evaluate the atrophy, white matter hyperintensities (WMHs), basal ganglia infarct (BGI), and infratentorial infarct (ITI) together. Our aim of this study is to propose a practical and standardized MRI for the clinicians to be used in daily practice. Methods: A total of 97 patients older than 60 years and diagnosed with depression or Alzheimer's disease (AD) are included. Cranial MRI, Mini Mental State Examination (MMSE), detailed neuropsychometric tests, and depression scales are applied to all patients. The SA, ventricular atrophy (VA), medial temporal lobe atrophy (MTA), periventricular WMH (PWMH), subcortical WMH (SCWMH), BGI, and ITI are scored according to the scale. The total score is also recorded. Results: The average age of the patients was 74.53 , and the mean MMSE score was 22.7 in the degenerative group and 27.8 in the non-degenerative group. Among the patients, 50 were diagnosed with AD. All parameters significantly increased with age. In the degenerative group, SA, VA, MTA, PWMH, SCWMH, and total scores were found to be significantly higher. Sensitivities of VA, PWMH, SCWMH, and total scores, as well as both sensitivity and specificities of MTA score, were observed to be high. When they were combined, sensitivities and specificities were found to be high. Conclusion: The scale is observed to be predictive in discriminating degenerative and non-degenerative processes. This discrimination is important, particularly in depressive patients complaining of forgetfulness.
\end{abstract}

RÉSUMÉ: Modifier une échelle d'évaluation visuelle dans le cas de patients présentant des pertes de mémoire et soumis à une IRM. Contexte: Dans la mesure où les manifestations de déficience cognitive ont tendance à augmenter avec le vieillissement, on constate aussi une augmentation de l'atrophie des sillons du cortex cérébral et de l'élargissement des ventricules cérébraux. En tenant compte des mesures propres à des échelles visuelles utilisées antérieurement, cette étude entend proposer une nouvelle échelle nous permettant d'évaluer en même temps des cas d'atrophie ainsi que la présence d'hyperdensités de la substance blanche, d'anomalies des ganglions de la base et d'infarctus affectant l'étage sus-tentoriel (infratentorial infarcts). L'objectif de cette étude est donc de proposer un examen d'IRM pratique et standardisé pouvant être utilisé quotidiennement par les cliniciens. Méthodes: Nous avons inclus dans cette étude 97 patients âgés de plus de 60 ans qui étaient soit atteints de dépression, soit de la maladie d'Alzheimer. Tous les patients recrutés ont été soumis à des examens d'IRM crâniens, au test de Folstein (ou MMSE), à un ensemble de tests neuro-psychométriques approfondis et à des échelles diagnostiques permettant d'évaluer la dépression. L'incidence de l'atrophie des sillons du cortex cérébral, de la région ventriculaire, du lobe temporal médian, des régions péri-ventriculaire et sous-corticale et de la substance blanche qu'elles contiennent, d'anomalies affectant les ganglions de base et d'infarctus à l'étage sus-tentoriel a été ainsi mesurée selon notre échelle. Le score total obtenu a aussi été enregistré. Résultats: L'âge moyen des patients était de 74,53 ans. Leur score moyen au test de Folstein était de 22,7 dans le cas du groupe de patients atteints d'une maladie dégénérative et de 27,8 dans le cas du groupe de patients n'étant pas atteints par ce type de maladie. Fait à noter, cinquante patients avaient reçu un diagnostic de maladie d'Alzheimer. Tous les paramètres évalués ont augmenté de façon notable avec l'âge. Ainsi, tant les scores obtenus dans le cas de l'atrophie des sillons du cortex cérébral, de celle affectant le lobe temporal médian, la région ventriculaire, la région péri-ventriculaire, la région sous-corticale que les scores totaux se sont révélés nettement plus élevés au sein du groupe de patients atteints d'une maladie dégénérative. La sensibilité des scores totaux et des scores évaluant l'atrophie des régions vasculaire, péri-vasculaire et sous-corticale, de même que la sensibilité et la spécificité des scores évaluant l'atrophie du lobe temporal médian, se sont révélées élevées. Lorsque combinées, la sensibilité et la spécificité sont apparues élevées. Conclusions: Notre échelle possède un caractère prédictif en ce qu'elle permet d'établir une distinction entre les processus dégénératifs et les processus nondégénératifs. Cette capacité est particulièrement importante dans le cas de patients dépressifs qui se plaignent de perte de mémoire.

From the Department of Neurology, Bayindir Icerenkoy Hospital, Atasehir, Istanbul, Turkey (BZY, MK); Department of Clinical Psychology, Bayindir Icerenkoy Hospital, Atasehir,

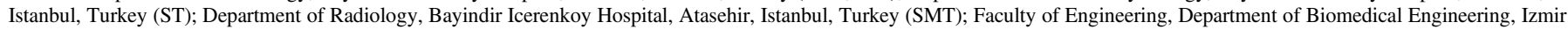
University of Economics, Balcova, Izmir, Turkey (DU).

Received January 25, 2017. Final Revisions Submitted May 18, 2018. Date of Acceptance August 2, 2018.

Correspondence to: M. Kandemir, Department of Neurology, Bayindir Icerenoy Hospital, Ali Nihat Tarlan Cad. Ertas Sok. No: 17, Atasehir 34752, Istanbul, Turkey.

Email: melekkandemir@gmail.com 
Keywords: Alzheimer, Dementia, Depression, Forgetfulness, Medial temporal lobe atrophy, Sulcal atrophy, Ventricular atrophy, Visual rating scale, White matter hyperintensity

doi:10.1017/cjn.2018.333

Can J Neurol Sci. 2019; 46: 71-78

\section{INTRODUCTION}

Patients complaining of forgetfulness admitted to the neurology outpatient clinics span across all age groups, but this rate increases significantly with age. Memory problems sometimes manifest as a degenerative process, or a symptom of treatable diseases such as depression. Brain imaging helps us not only exclude the reasons that might cause symptoms of dementia such as tumors and vascular lesions but also helps distinguish the type of dementia with degenerative nature. Although the volumetric and functional MRI and brain network studies have increased in recent years, conventional MRI reserves its importance in clinical practice. $^{1}$

It is known that cortical folding decreases with cognitive decline, whereas sulcal distance and ventricular enlargement increase. ${ }^{2-6}$ Several studies have shown that enlargement of the ventricles and the sulci were reliable indicators in detecting the neurodegenerative process; their increase was correlated with the progression of the disease, and they may be used in early diagnosis. ${ }^{3-6}$ Structural and functional imaging studies focusing on the medial temporal regionimportant for memory-have shown that the presence of medial temporal lobe atrophy (MTA) was predictive of the development of Alzheimer's disease (AD). ${ }^{7}$

The incidence of not only atrophy but also white matter hyperintensities (WMHs) increases with age, ${ }^{2,8-11}$ and may have negative effects on walking-balance disorders and mood, as well as cognitive functions. ${ }^{9,11-14}$ White matter hyperintensity has been defined in healthy subjects and in patients with vascular dementia (VD) and AD. ${ }^{8,10,15}$ Radiologically defined as focal or diffuse bright areas on T2 and fluid-attenuated inversion recovery (FLAIR) images, WMHs were often divided into two categories: (1) periventricular WMH (PWMH), located adjacent to the ventricles, and (2) subcortical WMH (SCWMH), which is observed as patchy regions in the subcortical white matter beyond the periventricular area. ${ }^{16-18}$ Increased signal intensity in the periventricular area, known as "bands" and "caps," can be seen in various diseases such as VD, Lewy body dementia (LBD), and AD and also in the case of normal aging. ${ }^{10,19}$ The hyperintensities of the basal ganglia and infratentorial areas, mostly associated with ischemic and gliotic changes, were more common in VD. ${ }^{10,16}$

Various scales have been developed to provide objective assessment of atrophic changes and $\mathrm{WMH}$, determine their severity, and enable their clinical use..$^{2,15,16,20,21}$ Visual grading scales are predominantly based on the identification of changes in the cerebral tissue with the naked eye and the atrophy and WMH by qualitative or semi-quantitative means. ${ }^{2,15,16,20}$ Temporal and frontal lobe atrophies and enlargement of the sulci and ventricles are used to evaluate the atrophy. ${ }^{2,22,23}$ At first, simple assessment of the existence of white matter changes was carried out-without distinction between periventricular and deep white matterwhereas in time more elaborate scales evaluating the periventricular and deep white matter areas as separate regions were developed. ${ }^{15,16,21,24}$ Basal ganglia hyperintensities are assessed separately in some scales, ${ }^{8,16,24}$ or together with infratentorial hyperintensities in others. ${ }^{2,16,24}$ The scale developed by Fazekas et al was confirmed histopathologically as well. ${ }^{19}$

Taking the measurements in the previously proposed visual scales into account, here we propose a new scale called "Modified Visual MRI Rating Scale (MVMRS)," which allows us to evaluate atrophy, WMH, basal ganglia infarct (BGI), and infratentorial infarct (ITI) together. The role of this scale in assessing MRI scans of patients with forgetfulness was investigated to differentiate between degenerative and non-degenerative (ND) processes. Our aim in this study was to propose a practical and standardized MRI scale that could easily be used by the clinicians in daily practice for diagnosis of degenerative processes and follow-up of patients' progress.

\section{Materials ANd Methods}

A total of 97 patients aged 60 and above were included in this study. Among them, 47 (48.5\%) patients were diagnosed with depression (ND group), whereas $50(51.5 \%)$ patients were diagnosed with $\mathrm{AD}$ (degenerative group) according to the Diagnostic and Statistical Manual of Mental Disorder-IV-Text Revision and NINCDS-ADRDA criteria, neurological and psychiatric examination, laboratory tests, cranial MRI, activities of daily living, and a detailed neuropsychological evaluation including the Mini Mental State Examination (MMSE) and the geriatric depression scale (GDS). The neuropsychological evaluation comprises a standard battery of psychometric tests that assess various cognitive functions such as attention, executive functions, language, verbal memory, and visiospatial abilities. Clinical dementia rating (CDR) scale was used to evaluate patients' daily functions.

The study was approved by our scientific, medical ethics, and deontology board, and all participants or their relatives gave informed consent.

MRIs of all patients were acquired with a $1.5 \mathrm{~T}$ Siemens Magnetom Avanto (Germany) scanner. MRIs were reviewed by two neurologists and one radiologist, and visually scored according to the MVMRS criteria by consensus agreement. Anomalies such as tumors noticed on MRIs were also noted. The proposed scale was created by considering multiple sets of criteria: more specifically, Yue et al for sulcal and ventricular atrophy; Scheltens et al for MTA; and those of Fazekas et al, Scheltens et al, and Erkinjuntti et al for periventricular, subcortical and deep white matter, basal ganglia, and infratentorial lesions. ${ }^{2,8,15,16,20}$

Sulcal atrophy (SA) was graded in a 0-9 range based on the widths of the central sulci, interhemispheric fissure, and other cortical sulci on the axial T1 (repetition time [TR] $=673 \mathrm{~ms}$, echo time $[\mathrm{TE}]=12 \mathrm{~ms}, 3$ or $5 \mathrm{~mm}$ slice thickness, $0.6 \mathrm{~mm}$ gap) image corresponding to the slice level where central sulci is best viewed (most similar to the shape of inverted omega).

Ventricular atrophy (VA) was graded in a 0-9 range on the axial $\mathrm{T} 1(\mathrm{TR}=673 \mathrm{~ms}, \mathrm{TE}=12 \mathrm{~ms}, 3$ or $5 \mathrm{~mm}$ slice thickness, $0.6 \mathrm{~mm}$ gap) slice showing the frontal and occipital horns of the lateral ventricles together with the third ventricle. 
Table 1: Details of the modified visual MRI rating scale

\begin{tabular}{|c|c|c|c|}
\hline & Sulcal & \multicolumn{2}{|l|}{$0-9$} \\
\hline Atrophy & Ventricular & \multicolumn{2}{|l|}{$0-9$} \\
\hline MTA & Width of choroid fissure & Width of temporal horn & $\begin{array}{l}\text { Height of hippocampal } \\
\text { formation }\end{array}$ \\
\hline 0 & $\mathrm{~N}$ & $\mathrm{~N}$ & $\mathrm{~N}$ \\
\hline 1 & $\uparrow$ & $\mathrm{N}$ & $\mathrm{N}$ \\
\hline 2 & $\uparrow \uparrow$ & $\uparrow$ & $\downarrow$ \\
\hline 3 & $\uparrow \uparrow \uparrow$ & $\uparrow \uparrow$ & $\downarrow \downarrow$ \\
\hline 4 & $\uparrow \uparrow \uparrow$ & $\uparrow \uparrow \uparrow$ & $\downarrow \downarrow \downarrow$ \\
\hline \multirow[t]{6}{*}{ WMH } & Periventricular & Subcortical & \\
\hline & 0 : No lesion & 0: No lesion & \\
\hline & 1: Caps & 1: $<5$ small focal and/or $<2$ large focal lesions & \\
\hline & 2: Thin line & 2: 5-12 small focal and/or 2-4 large focal lesions & \\
\hline & 3: Halo & 3: $>12$ small focal and/or $>4$ large focal or confluent lesions & \\
\hline & 4: Irregular, extending to the deep white matter & 4: Predominantly confluent lesions & \\
\hline \multirow[t]{4}{*}{ Infarcts } & Basal ganglia & Infratentorial & \\
\hline & 0: No lesion & 0: No lesion & \\
\hline & 1: Few lesions $(1-3)$ & 1: Few lesions $(1-3)$ & \\
\hline & 2: Many lesions $(>4)$ & 2: Many lesions (>4) & \\
\hline Other & Tumor etc. & & \\
\hline
\end{tabular}

$\mathrm{MRI}=$ magnetic resonance imaging; $\mathrm{MTA}=$ medial temporal lobe atrophy; $\mathrm{WMH}=$ White matter hyperintensities

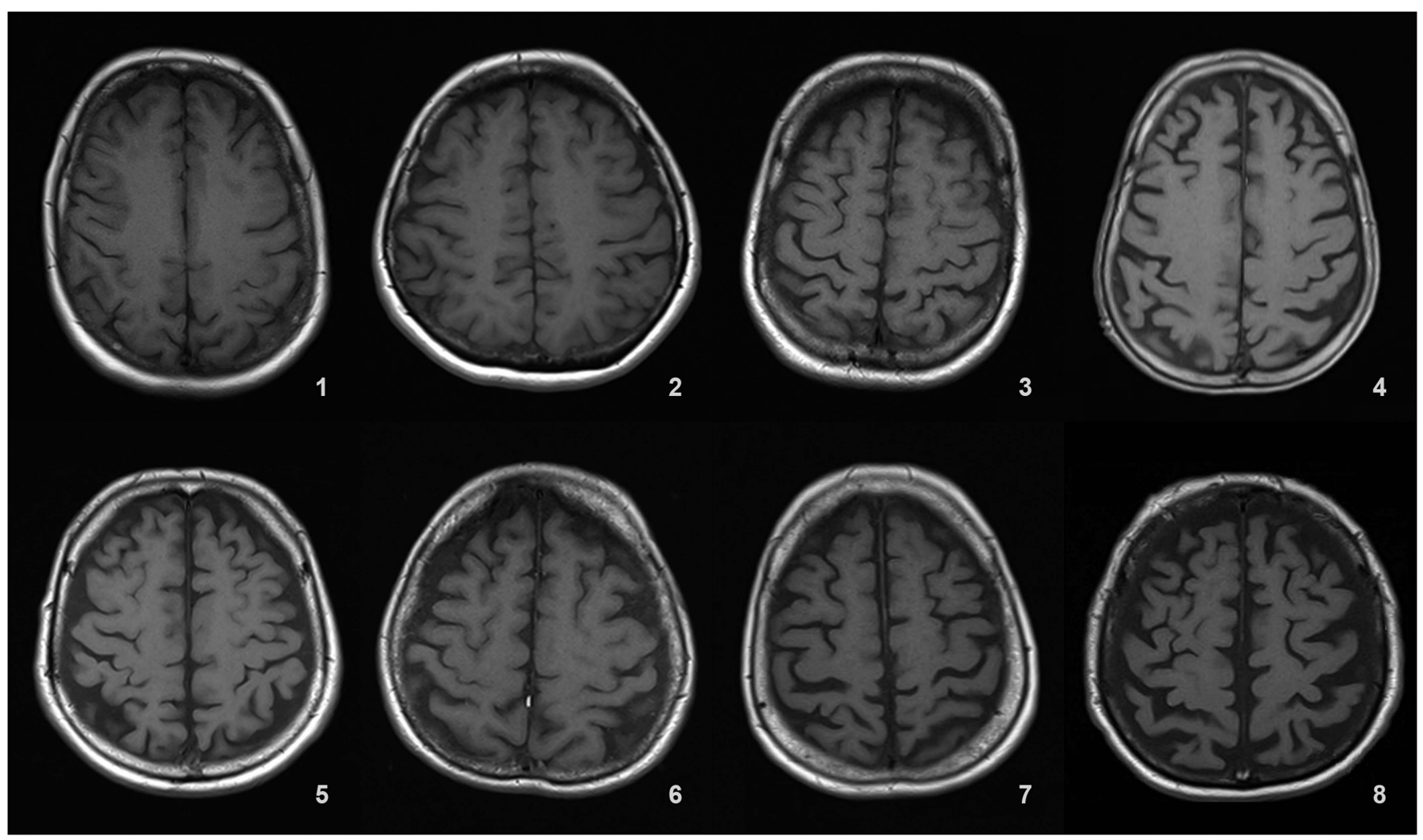

Figure 1: Reference MRIs corresponding to sulcal atrophy grades between 1-8. 


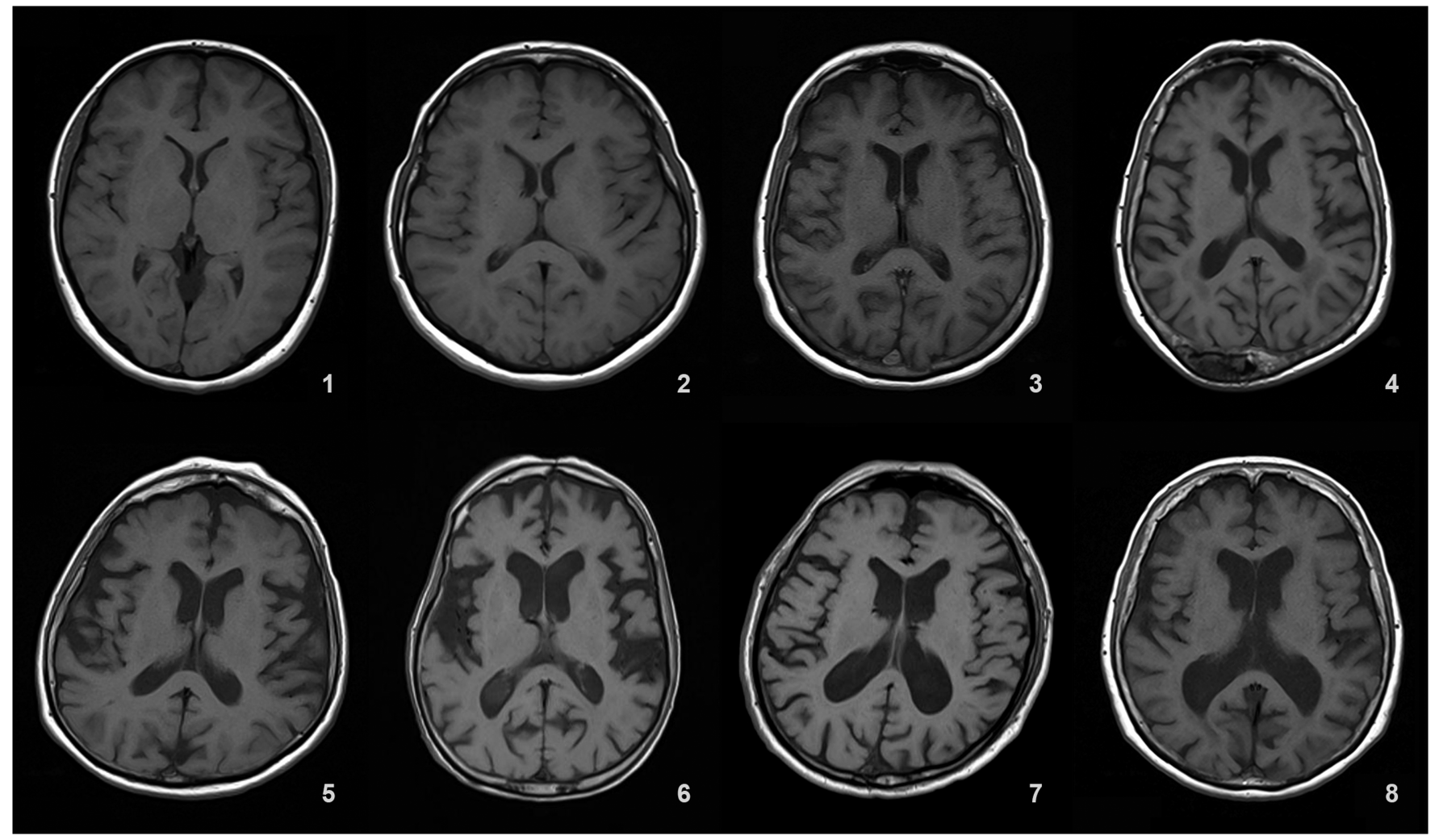

Figure 2: Reference MRIs corresponding to ventricular atrophy grades between 1-8.

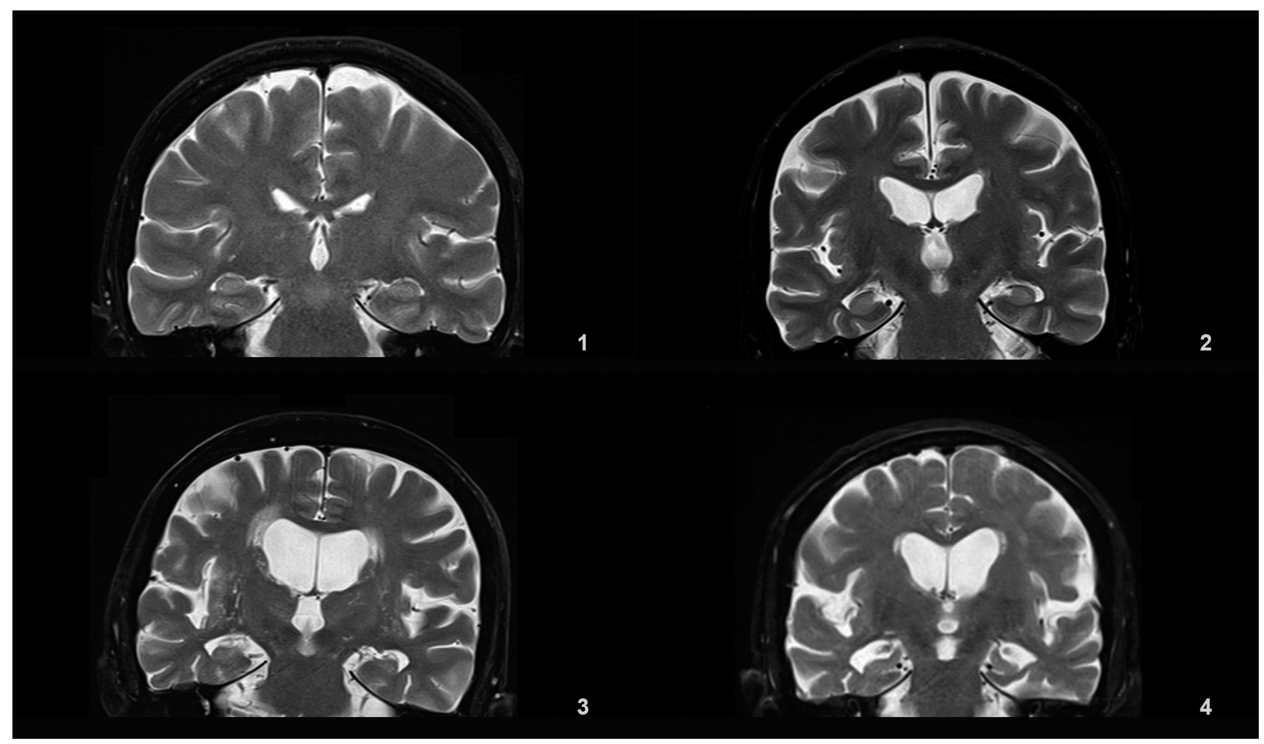

Figure 3: Reference MRIs corresponding to medial temporal atrophy grades between 1-4.

Medial temporal lobe atrophy was graded in a 0-4 range based on the widths of the choroid fissure and temporal horn, and the height of the hippocampal formation on the coronal $\mathrm{T} 2(\mathrm{TR}=$ $6000 \mathrm{~ms}, \mathrm{TE}=90 \mathrm{~ms}, 3$ or $5 \mathrm{~mm}$ slice thickness, $0.9 \mathrm{~mm}$ gap) slice through the corpus of the hippocampus (at the level of the anterior pons).

Periventricular WMH was graded in a 0-4 range based on the $\mathrm{WMH}$ identified as continuous, confluent areas of high signal intensity adjacent to anterior or posterior horns of the lateral ventricles and along the ventricular system on FLAIR $(\mathrm{TR}=$ $8000 \mathrm{~ms}, \mathrm{TE}=80 \mathrm{~ms}, 3$ or $5 \mathrm{~mm}$ slice thickness, $0.6 \mathrm{~mm}$ gap) images.

Subcortical WMH was graded in a 0-4 range based on the WMH identified as lesions located in the white matter but not touching the periventricular area on FLAIR $(\mathrm{TR}=8000 \mathrm{~ms}$, $\mathrm{TE}=80 \mathrm{~ms}, 3$ or $5 \mathrm{~mm}$ slice thickness, $0.6 \mathrm{~mm}$ gap) images. 


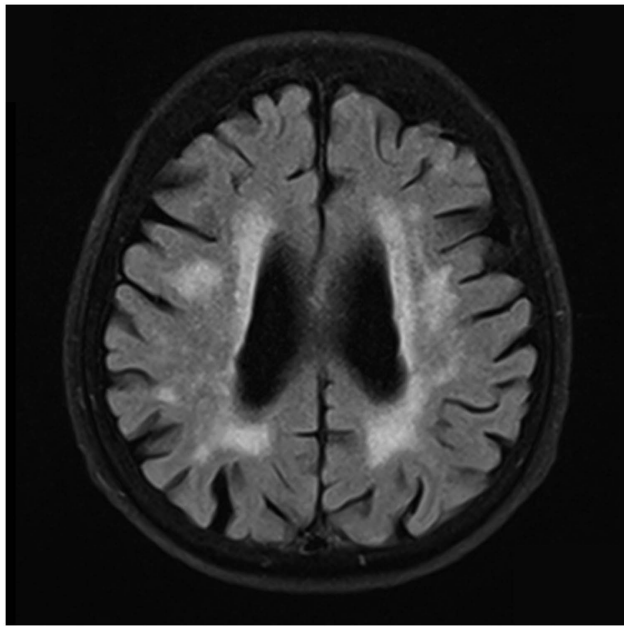

Figure 4: $M R I$ of a patient with $P W M H=4$ and $S C W M H=3$.

Table 2: Demographic features of the patients

\begin{tabular}{l|c|c|c|c}
\hline & \multicolumn{2}{|l|}{ AD $(\boldsymbol{n}=\mathbf{5 0})$} & \multicolumn{2}{c}{ Depression $(\boldsymbol{n}=\mathbf{4 7})$} \\
\hline & Range & Mean \pm SD & & Mean \pm SD \\
\hline Age (years) & $63-99$ & $78.92 \pm 6.37$ & $60-86$ & $69.85 \pm 6.61$ \\
\hline Gender & \multicolumn{5}{|l|}{} \\
\hline Female, $n(\%)$ & $20(40 \%)$ & $30(63.8 \%)$ \\
\hline Male, $n(\%)$ & $30(60 \%)$ & $17(36.2 \%)$ \\
\hline Education (years) & $0-18$ & $9.40 \pm 4.50$ & $0-15$ & $10.28 \pm 3.82$ \\
\hline Duration of complaints (years) & $0-10$ & $1.78 \pm 1.98$ & $0.1-17$ & $2.68 \pm 3.59$ \\
\hline MMSE & $7-29$ & $22.78 \pm 6.03$ & $20-30$ & $27.83 \pm 2.26$ \\
\hline GDS & $0-22$ & $9.38 \pm 5.30$ & $1-23$ & $11.59 \pm 5.74$ \\
\hline CDR & $0.5-3$ & $1.15 \pm 0.66$ & & \\
\hline
\end{tabular}

$\mathrm{AD}=$ Alzheimer's disease $; \mathrm{CDR}=$ Clinical dementia rating scale; GDS $=$ Geriatric depression scale; MMSE $=$ Mini Mental State Examination; $\mathrm{SD}=$ standard deviation

Basal ganglia infarcts were graded in a 0-2 range from FLAIR $(\mathrm{TR}=8000 \mathrm{~ms}, \mathrm{TE}=80 \mathrm{~ms}, 3$ or $5 \mathrm{~mm}$ slice thickness, $0.6 \mathrm{~mm}$ gap) based on the hyperintensities in caudat nucleus, putamen, globus pallidus, and thalamus regions.

Infratentorial infarcts were graded in a 0-2 range from FLAIR $(\mathrm{TR}=8000 \mathrm{~ms}, \mathrm{TE}=80 \mathrm{~ms}, 3$ or $5 \mathrm{~mm}$ slice thickness, $0.6 \mathrm{~mm}$ gap) based on the hyperintensities in brainstem and cerebellum.

Details of the proposed scale are given in Table 1. For grading the SA, VA, and MTA, a reference data set was created from cranial MRIs of 30 subjects who were admitted to the clinic owing to complaints other than forgetfulness, but they were not included in the study (Figures 1-3). An exemplary MRI scan of a patient graded with PWMH $=4$ and SCWMH $=3$ can be seen in Figure 4.

\section{Statistical Analyses}

The statistical analyses were performed using Statistical Package for Social Sciences for Windows 23.0. Numeric variables of groups were assessed with the Kruskal-Wallis $H$-test and Mann-Whitney $U$-test. Categoric variables were assessed with the
Table 3: Documentation of the patients' scores of the sulcal/ ventricular atrophy, medial temporal lobe atrophy, white matter hyperintensities, and total

\begin{tabular}{l|c|c|c|c|c}
\hline & $\begin{array}{l}\text { Min- } \\
\text { Max }\end{array}$ & $\begin{array}{l}\text { AD }(\boldsymbol{n}=\mathbf{5 0}) \\
\text { mean } \pm \text { SD }\end{array}$ & $\begin{array}{l}\text { Depression } \\
(\boldsymbol{n}=\mathbf{4 7}) \\
\text { mean } \pm \text { SD }\end{array}$ & MW $^{\dagger}$ & $p^{*}$ \\
\hline SAs & $1-9$ & $6.08 \pm 1.82$ & $4.70 \pm 2.17$ & 736.000 & 0.001 \\
\hline VAs & $1-8$ & $4.92 \pm 1.65$ & $3.15 \pm 1.46$ & 491.000 & $<0.0001$ \\
\hline MTAs & $0-4$ & $2.34 \pm 0.94$ & $1.17 \pm 0.64$ & 394.000 & $<0.0001$ \\
\hline PWMHs & $0-4$ & $1.52 \pm 1.23$ & $1.02 \pm 0.92$ & 911.000 & 0.048 \\
\hline SCWMHs & $0-4$ & $2.22 \pm 1.06$ & $1.66 \pm 1.17$ & 851.000 & 0.015 \\
\hline BGIs & $0-2$ & $0.16 \pm 0.42$ & $0.17 \pm 0.43$ & 1164.500 & 0.901 \\
\hline ITIs & $0-2$ & $0.14 \pm 0.40$ & $0.20 \pm 0.15$ & 1058.500 & 0.061 \\
\hline Ts & $5-26$ & $17.38 \pm 4.40$ & $11.89 \pm 4.12$ & 415.000 & $<0.0001$ \\
\hline
\end{tabular}

${ }^{*} p<0.05$ is considered statistically significant. ${ }^{\dagger}$ Mann-Whitney $U$-test $\mathrm{AD}=$ Alzheimer's disease; BGIs = basal ganglia infarct score; ITIs = infratentorial infarct score; MTAs = medial temporal lobe atrophy score; PWMHs = periventricular white matter hyperintensity score; SAs = sulcal atrophy score; SCWMHs = subcortical white matter hyperintensity score; $\mathrm{SD}=$ standard deviation; $\mathrm{Ts}=$ total score; VAs = ventricular atrophy score

Table 4: Receiver operating characteristic analysis results of the MVMRS parameters considered individually

\begin{tabular}{l|c|c|c|c|c}
\hline & Cutoff & \multicolumn{1}{|c|}{ AUC } & \multicolumn{1}{l|}{$\boldsymbol{p}^{*}$} & Sensitivity (\%) & Specificity (\%) \\
\hline SAs & $>6$ & 0.687 & 0.0006 & 64 & 66 \\
\hline VAs & $>6$ & 0.791 & $<0.0001$ & 91 & 44 \\
\hline MTAs & $>2$ & 0.832 & $<0.0001$ & 79 & 80 \\
\hline PWMHs & $>3$ & 0.612 & 0.050 & 96 & 24 \\
\hline SCWMHs & $>3$ & 0.638 & 0.0145 & 72 & 50 \\
\hline Ts & $>16$ & 0.823 & $<0.0001$ & 81 & 64 \\
\hline
\end{tabular}

$* p<0.05$ is considered statistically significant.

AUC $=$ area under the curve; MTAs = medial temporal lobe atrophy score; $\mathrm{MVMRS}=$ modified visual MRI rating scale; $\mathrm{PWMHs}=$ periventricular white matter hyperintensity score; SAs = sulcal atrophy score; SCWMHs = subcortical white matter hyperintensity score; Ts = total score; VAs = ventricular atrophy score

$\chi^{2}$-test. The differences between AD and ND groups were assessed with the Mann-Whitney $U$-test and $\chi^{2}$-test. Cutoff values for the measurements of ND and degenerative processes were determined based on receiver operating characteristic curve analysis. The measurements were categorized using the cutoff values, and sensitivity and specificity were calculated for their combinations. $p<0.05$ was considered statistically significant.

\section{RESULTS}

Demographic features of the patients are given in Table 2. Our mean scores for the MMSE and GDS indicate mild cognitive impairment (MCI) and mild depression because of some patients' high level of education and cognitive reserve, and subtle depressive findings, which they did not mention in scales. Table 3 shows 
Table 5: Receiver operating characteristic analysis results of the MVMRS parameters in dual combinations

\begin{tabular}{l|c|c}
\hline & Sensitivity (\%) & Specificity (\%) \\
\hline SAs + PWMHs & 62 & 72 \\
\hline SAs + SCWMHs & 49 & 82 \\
\hline VAs + PWMHs & 89 & 56 \\
\hline VAs + SCWMHs & 68 & 70 \\
\hline MTAs + PWMHs & 77 & 82 \\
\hline MTAs + SCWMHs & 57 & 86 \\
\hline MTAs + VAs & 77 & 82 \\
\hline
\end{tabular}

MTAs = medial temporal lobe atrophy score; MVMRS = modified visual MRI rating scale; $\mathrm{PWMHs}=$ periventricular white matter hyperintensity score; SAs = sulcal atrophy score; SCWMHs = subcortical white matter hyperintensity score; $\mathrm{Ts}=$ total score; VAs = ventricular atrophy score

SA, VA, MTA, PWMH, SCWMH, BGI, ITI, and total scores of all patients according to the MVMRS scale. The SA score (SAs) $(p=0.001)$, VA score (VAs) $(p<0.0001)$, MTA score (MTAs) $(p<0.0001)$, PWMH score (PWMHs) $(p=0.048)$, SCWMH score (SCWMHs) $(p=0.015)$, and total score (Ts) $(p<0.0001)$ in the degenerative group are significantly higher than those in the ND group (Table 3). The SAs, VAs, MTAs, PWMHs, SCWMHs, and Ts are found to be increasing significantly with age (Supplementary Table 1$)$. The SAs $(p=0.002)$, VAs $(p<0.0001)$, MTAs $(p=0.001)$, and Ts $(p<0.0001)$ are significantly higher in males (Supplementary Table 2). There is no significant relationship between the parameters and the level of education and the duration of complaint. The results show nine patients $(9.3 \%)$ have a family history of dementia. Forgetfulness is the only complaint for $34(72.3 \%)$ subjects with depression and 37 (74\%) subjects with AD. In 26 patients (26.8\%), other complaints such as word finding difficulties $(6.2 \%)$, attention deficits $(7.2 \%)$, walking and balance impairment $(9.3 \%)$, depressive symptoms $(16.5 \%)$, and hallucinations $(5.2 \%)$ are documented. Only $21.3 \%$ of the patients in the ND group present typical depressive complaints accompanied by forgetfulness. The VAs $(p=0.022)$, PWMHs $(p=0.009)$, and Ts $(p=0.009)$ are found to be significantly lower in patients with attention deficits; SAs $(p=0.037)$, VAs $(p=0.009)$, and Ts $(p=0.008)$ are significantly higher in patients with walking and balance impairment; BGI scores (BGIs) $(p=0.026)$ are significantly higher in those displaying depressive symptoms (Supplementary Table 3).

Regarding the parameters considered in the scale and found to be significant, the cutoff values are determined as $>6$ for SAs, $>6$ for VAs, $>2$ for MTAs, $>3$ for PWMHs, $>3$ for SCWMHs, and $>16$ for Ts. The sensitivities of VAs, PWMHs, SCWMHs, and Ts, and both sensitivity and specificity of MTAs, are high. When viewed in combination, sensitivities of VAs + PWMHs, VAs + SCWMHs, VAs + MTAs, and MTAs + PWMHs and the specificities of SAs + PWMHs, SAs + SCWMHs, VAs + SCWMHs, MTAs + PWMHs, MTAs + SCWMHs, and VAs + MTAs are high. Details are given in Tables 4 and 5.

There is a negative correlation between MMSE scores and SAs $(p=0.011)$, VAs $(p=0.004)$, MTAs $(p=0.001)$, SCWMHs $(p=0.032)$, and Ts $(p<0.0001)$. A positive correlation is observed between CDR and MTAs ( $p=0.035)$, PWMHs $(p=0.022)$, and Ts $(p=0.045)$ (Supplementary Table 4). EEG could be performed in 53 $(54.6 \%)$ patients, where 15 of them $(15.4 \%)$ showed disorganization in baseline rhythms. The VAs $(p=0.022)$ and MTAs $(p=0.020)$ are found to be significantly higher in patients with disorganized bioelectrical activity seen on EEG (Supplementary Table 5).

\section{DISCUSSION}

Forgetfulness is a very common symptom in neurology clinical practice among all ages. Most of the patients who complain about forgetfulness have a degenerative disease or depression. As each require different treatment and follow-up strategies, we preferred to take $\mathrm{AD}$ - the most common cause of degenerative dementiato represent the degenerative group, and depression-the most common ND cause of forgetfulness-for the ND group.

By using MVMRS for screening, MRIs of patients admitted to the neurology clinic with complaint of forgetfulness, SAs, VAs, MTAs, PWMHs, SCWMHs, and Ts are found to be significant in discriminating degenerative and ND processes. The observations of high sensitivity for VAs, PWMHs, SCWMHs, and high sensitivity and specificity for MTAs are consistent with other studies. ${ }^{2,4,11,20}$ The high sensitivity of Ts, which has not been evaluated in previous studies, supports that the assessment of this scale as a whole is also informative.

The combined assessment of MTAs with VAs, SCWMHs, and PWMHs is found to be more meaningful than their individual evaluation, indicating that the combined assessment is beneficial in identifying the degenerative process. The fact that the combined evaluation of SAs and SCWMHs is more specific than their individual evaluations supports the findings that subcortical lesions increase the risk of cognitive impairment. ${ }^{11,25,26}$

MRI grading scales have already been developed to demonstrate the relationship between the signs of cognitive impairment observed in elderly - forgetfulness being at the forefront-and cerebral atrophy and white matter changes. ${ }^{2,8,15,20}$ An ideal scale should allow for grading of both $\mathrm{WMH}$ and atrophic changes in a standardized and practical manner suitable for clinical use. In previous studies, atrophy has been evaluated mainly in the temporal and frontal regions, whereas the widths of the central sulci and lateral ventricles have also been exploited. ${ }^{2,22,23}$ It has been shown that in addition to the cortical sulci atrophy the evaluation of interhemispheric atrophy is also reliable. ${ }^{27}$ In addition to SA, ventricular enlargement has been proposed as a sensitive and objective marker in identifying the degenerative process, and it has been reported to negatively affect the cognitive performance in elderly without dementia., ${ }^{2,22}$ It has been shown that MTA, VA, and SA increase over time in AD patients, ${ }^{3,28}$ whereas SA and VA better correlate with changes in neuropsychometric tests than MTA. ${ }^{3}$ In our study, especially high VAs, MTAs, and Ts are also found to be significant in terms of degenerative process. It has been observed that MMSE scores decreased as sulcal and ventricular enlargement increased. ${ }^{6,22}$ In our study, it is unsurprisingly observed that the increase in SAs, VAs, MTAs, and Ts and the decrease in MMSE scores show a significant correlation, and that MTAs and Ts increase significantly in correlation with the CDR score.

There are several verified and commonly used rating scales for visual scoring of WMH. ${ }^{8,15,16,21}$ Similar to our study, in some of these scales frontal and occipital "caps" or pencil-thin linings are considered normal. ${ }^{10,29}$ It has been shown that occipital "caps" are more common in all dementia groups (VD, AD, and LBD), whereas periventricular bands are more severe in $\mathrm{AD}$ and $\mathrm{VD}$ cases. ${ }^{10}$ 
Although it is believed that PWMH better reflects total WMH burden, and PWMH and SCWMH are intercorrelated processes sharing the same etiology that may even have contiguous localization, their independent evaluations may be arbitrary. ${ }^{18}$ Periventricular bands and caps have been found to be caused by demyelination related to loss of ependymal lining and subependymal gliosis, whereas punctate and confluent lesions are due to ischemic tissue damage. ${ }^{19}$ Scheltens et $\mathrm{al}^{16,21}$ and Erkinjuntti et $\mathrm{al}^{8}$ included hyperintensities in the basal ganglia, and Scheltens et $\mathrm{al}^{16}$ included infratentorial hyperintensities in their scales, and suggested that such hyperintensities are evidence of small vessel diseases.

The prevalence, severity, and distribution of WMH in both healthy older adults and those with dementia are strongly correlated with vascular risk factors and age. ${ }^{10,11,17}$ The imaging studies demonstrated that severe WMH is associated with cognitive dysfunction, increases the risk of developing dementia and AD in healthy population, and has greater combined effect with MTA and degenerative process on cognition. ${ }^{11,18,26,30}$ The relation between WMH load and cognitive impairment, especially executive functions, speed of information processing, and depressive symptoms, in elderly people without dementia has also been reported. ${ }^{11-14,17,25,31}$ Although a negative correlation between MMSE scores and SCWMHs is observed in our study, there is no correlation with PWMHs, which is consistent with the finding of Barber et al. ${ }^{10}$ It is noticed that the higher the CDR score, the more frequent the PWMHs. Some researchers have stated that they detected the negative effect of PWMH but not SCWMH on cognitive function; however, they did not completely exclude the effect of subcortical lesions as they may have gone unnoticed owing to the tests used. ${ }^{17}$

In our study, a large proportion of both degenerative and ND group patients have forgetfulness complaints alone, whereas a small number of depressed patients have typical depressive complaints accompanied by forgetfulness. The fact that the proportion of patients with depressive symptoms in both groups is very low suggests that forgetfulness may conceal typical depressive complaints such as unhappiness, reluctance, and anergy in the ND group as well. The fact that all patients with complaints of attention deficit accompanying forgetfulness are from the ND group and that they present low Ts suggests that depressive patients often express the symptom of "forgetfulness and attention deficit" as a complaint. Non-cognitive complaints such as walking and balance disorders may also be associated with $\mathrm{WMH},{ }^{9,14}$ which is explained by the increase in WMH that affects the descending and ascending pathways in the periventricular region. ${ }^{9}$ Although a relationship between walking and balance impairments and WMH has not been detected in our study, it has been observed that SAs, VAs, and Ts are higher in these patients.

Cerebrovascular diseases, VD, and AD are frequently seen in the same age group and have common risk factors. It is stated in the literature that these abnormalities are less frequent in basal ganglia and infratentorial regions in healthy controls and $\mathrm{AD},{ }^{2,16}$ but in patients with VD basal ganglia lesions are more common and may assist in differential diagnosis. ${ }^{10}$ In MCI patients, basal ganglia or infratentrorial hyperintensities have been less frequently observed and have not been associated with cognitive parameters. ${ }^{13,25}$ In recent years, depression in the elderly has been reported more frequently in patients with silent BGIs, especially those with left hemisphere localization, but no association with silent infratentorial infarcts has been detected. ${ }^{32}$ In our study, BGI are detected in a very small number of patients and there is no difference between the two groups in this respect. However, it is noteworthy that most of the identified BGI are bilateral and that depressive symptoms are more common in these patients.

Inconsistent with the literature, we observe that sulcal and ventricular enlargement together with the MTA and the prevalence and severity of WMH increase with age, and they appear more severely in AD patients. ${ }^{8,10,11,17,29}$ Unlike other studies, there is no significant difference between the two genders in terms of WMH, ${ }^{2,17}$ although SAs, VAs, MTAs, and Ts are found to be higher in males compared with females, which supports the findings of Yue et al. ${ }^{2}$

\section{CONCLUSIONS}

MVMRS, which we recommend for the standardization of MRI evaluation in examination of patients with forgetfulness, can assess both atrophy and white matter damage, as well as MTA and infarcts, in the basal ganglia and infratentorial regions. This scale gives us the opportunity to evaluate such parameters in different combinations and as a whole. We would like to emphasize the importance of distinguishing depression, a common problem in the elderly, from a degenerative process, and the standard assessment of MRI in this respect. Although our data set is short in hyperintensities in the basal ganglia and infratentorial regions, we think that the relationship between depressive symptoms and basal ganglia lesions should be considered. In future studies, it would be useful to use MVMRS to evaluate wider groups of patients with different patterns of cognitive impairment and dementia subtypes.

\section{ACKNOWLEDGMENTS}

This work is partially supported by the Scientific and Technological Research Council of Turkey (TUBITAK) under grant no. 111E083. The authors thank Ceyda Afacan for the statistical analyses and Ishak Denizcan Ince for the translation.

Standardized reference image set used in this study is available for research purposes. Those interested may contact the corresponding author.

\section{Disclosures}

BZY, MK, SMT and DU report grants from Scientific and Technological Research Council of Turkey (TUBITAK), outside the submitted work. ST has nothing to disclose. Work was carried out at Bayindir Icerenkoy Hospital, Department of Neurology, 34752 Atasehir/Istanbul.

\section{SUPPlementary Materials}

To view supplementary material for this article, please visit https://doi.org/10.1017/cjn.2018.333

\section{REFERENCES}

1. Hafkemeijer A, van der Grond J, Rombouts SA. Imaging the default mode network in aging and dementia. Biochim Biophys Acta. 2012;1822(3):431-41.

2. Yue NC, Arnold AM, Longstreth WT, et al. Sulcal, ventricular, and white matter changes at MR imaging in the aging brain: data from the cardiovascular health study. Radiology. 1997;202(1): 33-9. 
3. Jack CR Jr, Shiung MM, Gunter JL, et al. Comparison of different MRI brain atrophy rate measures with clinical disease progression in AD. Neurology. 2004;62(4):591-600.

4. Nestor SM, Rupsingh R, Borrie M, et al. Ventricular enlargement as a possible measure of Alzheimer's disease progression validated using the Alzheimer's disease neuroimaging initiative database. Brain. 2008;131(Pt 9):2443-54.

5. Liu T, Wen W, Zhu W, et al. The relationship between cortical sulcal variability and cognitive performance in the elderly. Neuroimage. 2011;56(3):865-73.

6. Liu T, Lipnicki DM, Zhu W, et al. Cortical gyrification and sulcal spans in early stage Alzheimer's disease. PLoS One. 2012;7(2): e31083.

7. Visser PJ, Verhey FR, Hofman PA, et al. Medial temporal lobe atrophy predicts Alzheimer's disease in patients with minor cognitive impairment. J Neurol Neurosurg Psychiatry. 2002;72 (4):491-7.

8. Erkinjuntti T, Gao F, Lee DH, et al. Lack of difference in brain hyperintensities between patients with early Alzheimer's disease and control subjects. Arch Neurol. 1994;51(3):260-8.

9. Longstreth WT, Manolio TA, Arnold A, et al. Clinical correlates of white matter findings on cranial magnetic resonance imaging of 3301 elderly people. The Cardiovascular Health Study. Stroke. 1996;27(8):1274-82.

10. Barber R, Scheltens P, Gholkar A, et al. White matter lesions on magnetic resonance imaging in dementia with Lewy bodies, Alzheimer's disease, vascular dementia, and normal aging. J Neurol Neurosurg Psychiatry. 1999;67(1):66-72.

11. Debette S, Markus HS. The clinical importance of white matter hyperintensities on brain magnetic resonance imaging: systematic review and meta-analysis. BMJ. 2010;341:c3666.

12. Thomas AJ, Perry R, Kalaria RN, et al. Neuropathological evidence for ischemia in the white matter of the dorsolateral prefrontal cortex in late-life depression. Int J Geriatr Psychiatry. 2003;18 (1):7-13.

13. Debette S, Bombois S, Bruandet A, et al. Subcortical hyperintensities are associated with cognitive decline in patients with mild cognitive impairment. Stroke. 2007;38(11):2924-30.

14. Wardlaw JM, Valdés Hernández MC, Muñoz-Maniega S. What are white matter hyperintensities made of? Relevance to vascular cognitive impairment. J Am Heart Assoc. 2015;4(6):001140.

15. Fazekas F, Chawluk JB, Alavi A, et al. MR signal abnormalities at $1.5 \mathrm{~T}$ in Alzheimer's dementia and normal aging. AJR Am J Roentgenol. 1987;149(2):351-6.

16. Scheltens $P$, Barkhof $F$, Leys $D$, et al. A semiquantitative rating scale for the assessment of signal hyperintensities on magnetic resonance imaging. J Neurol Sci. 1993;114(1):7-12.

17. de Groot JC, de Leeuw FE, Oudkerk M, et al. Cerebral white matter lesions and cognitive function: The Rotterdam Scan Study. Ann Neurol. 2000;47(2):145-51.

18. DeCarli C, Fletcher E, Ramey V, et al. Anatomical mapping of white matter hyperintensities (WMH). Exploring the relationships between periventricular WMH, deep $\mathrm{WMH}$, and total WMH burden. Stroke. 2005;36(1):50-5.

19. Fazekas F, Kleinert R, Offenbacher H, et al. Pathologic correlates of incidental MRI white matter signal hyperintensities. Neurology. 1993;43(9):1683-9.

20. Scheltens P, Leys D, Barkhof F, et al. Atrophy of medial temporal lobes on MRI in "probable" Alzheimer's disease and normal ageing: diagnostic value and neuropsychological correlates. J Neurol Neurosurg Psychiatry. 1992;55(10):967-72.

21. Scheltens P, Erkinjunti T, Leys D, et al. White matter changes on CT and MRI: an overview of visual rating scales. European Task Force on age-related white matter changes. Eur Neurol. 1998;39 (2):80-9.

22. Breteler MM, van Amerongen NM, van Swieten JC, et al. Cognitive correlates of ventricular enlargement and cerebral white matter lesions on magnetic resonance imaging. The Rotterdam Study. Stroke. 1994;25(6):1109-15.

23. Whitwell JL, Peterson RC, Negash S, et al. Patterns of atrophy differ among specific subtypes of mild cognitive impairment. Arch Neurol. 2007;64(8):1130-8.

24. Wahlund LO, Barkhof F, Fazekas F, et al. A new rating scale for agerelated white matter changes applicable to MRI and CT. Stroke. 2001;32(6):1318-22.

25. Bombois S, Debette S, Delbeuck X, et al. Prevalence of subcortical vascular lesions and association with executive function in mild cognitive impairment subtypes. Stroke. 2007;38(9):2595-7.

26. Bombois S, Debette S, Bruandet A, et al. Vascular subcortical hyperintensities predict conversion to vascular and mixed dementia in MCI patients. Stroke. 2008;39(7):2046-51.

27. Baskaya O, Acar M, Kandemir M, et al. Inter-hemispheric atrophy better correlates with expert ratings than hemispheric cortical atrophy. SIU'12. 2012:1-4, https://doi.org/10.1109/SIU.2012. 6204783.

28. Iheme LO, Baskaya O, Sennaz A, et al. Atrophy measurement biomarkers using structural MRI for Alzheimer's disease. Workshop on novel imaging biomarkers for Alzheimer's Disease and related disorders (NIBAD) MICCAI'12, 2012.

29. Schmidt R, Fazekas F, Kleinert G, et al. Magnetic resonance imaging signal hyperintensities in the deep and subcortical white matter: a comparative study between stroke patients and normal volunteers. Arch Neurol. 1992;49(8):825-7.

30. van der Flier WM, van Straaten ECW, Barkhof F, et al. Medial temporal lobe atrophy and white matter hyperintensities are associated with mild cognitive deficits in non-disabled elderly people: The LADIS Study. J Neurol Neurosurg Psychiatry. 2005;76(11):1497-500.

31. van der Flier WM, van Straaten ECW, Barkhof F, et al. Small vessel disease and general cognitive function in nondisabled elderly. The LADIS Study. Stroke. 2005;36(10):2116-20.

32. Wu RH, Feng C, Xu Y, Hua T, et al. Late-onset depression in the absence of stroke: associated with silent brain infarctions, microbleeds and lesion locations. Int J Med Sci. 2014;11(6):587-92. 